\title{
Correction to: Impact of coronavirus disease-2019 on pediatric nephrology practice and education: an ESPN survey
}

\author{
Burcu Yazıcıoğlu ${ }^{1}$ - Sevcan A. Bakkaloğlu ${ }^{1}$ the European Society for Pediatric Nephrology \\ Published online: 24 February 2022 \\ (c) The Author(s), under exclusive licence to International Pediatric Nephrology Association 2022, corrected publication 2022
}

\author{
Correction to: Pediatr Nephrol \\ https://doi.org/10.1007/s00467-021-05226-1
}

The original version of this article unfortunately contained a mistake. During the process of typesetting, the list of participating collaborators from the European Society for Pediatric Nephrology cited in this article was not properly updated. The correct list of participating members for the here cited study is presented below. The published apologizes for this mistake. The original article was updated.

\section{ESPN Collaborators and Contributors Coordinated by the ESPN-supported Survey}

Abranches M, Centro Hospitalar e Universitário de Lisboa Central, Hospital Dona Estefânia, Unidade de Nefrologia Pediátrica, Lisboa, Portugal

Akman S, Department of Pediatric Nephrology, Akdeniz University Faculty of Medicine, Antalya, Turkey

Alpay H, Department of Pediatric Nephrology, Marmara University Faculty of Medicine, İstanbul, Turkey

Ariceta G, Hospital Vall d' Hebron, Universidad Autónoma de Barcelona, Spain

Atmış B, Department of Pediatric Nephrology, Çukurova University Faculty of Medicine, Adana, Turkey

Bael A, University of Antwerp, Faculty of Medicine, Antwerpen, Belgium

Bakkaloğlu SA, Department of Pediatric Nephrology, Gazi University Faculty of Medicine, Ankara, Turkey

The original article can be found online at https://doi.org/10.1007/ s00467-021-05226-1.

Sevcan A. Bakkaloğlu

sevcan@gazi.edu.tr

1 Department of Pediatric Nephrology, Gazi University School of Medicine, Ankara, Turkey
Bayrakçı US, Department of Pediatric Nephrology, Ankara City Hospital - Yıldırım Beyazıt University, Ankara, Turkey

Bhimma R, Department of Pediatrics and Child Health, College of Health Sciences, Nelson R Mandela School of Medicine, University of KwaZulu-Natal, Durban, South Africa Bjerre A, Department of Pediatrics, Oslo University Hospital, Oslo; Norway

Bonzel KE, Rehabilitation Center for Children and Youth Awaiting Organ Transplantation and Thereafter, Tirol, Austria

Çeleğen K, Department of Pediatric Nephrology, Afyonkarahisar State Hospital, Afyonkarahisar, Turkey

Delibaş A, Department of Pediatric Nephrology, Mersin University Faculty of Medicine, Mersin, Turkey

Demircioğlu B, Department of Pediatric Nephrology, Gaziantep University Faculty of Medicine, Gaziantep, Turkey

Dursun İ, Department of Pediatric Nephrology, Erciyes University Faculty of Medicine, Kayseri, Turkey

Ertan P, Department of Pediatric Nephrology, Celal Bayar University Faculty of Medicine, Manisa, Turkey

Flögelova H, Division of Nephrology, Department of Pediatrics, Palacky University Faculty of Medicine, Faculty Hospital in Olomouc, Czech Republic

Gülleroğlu K, Department of Pediatric Nephrology, Başkent University Faculty of Medicine, Ankara, Turkey

Gürgöze MK, Department of Pediatric Nephrology, Firat University Faculty of Medicine, Elazı $\breve{g}$, Turkey

Hacıhamdioğlu DÖ, Department of Pediatric Nephrology, Medical Park Hospital, İstanbul, Turkey

Haffner D, Department of Pediatric Kidney, Liver and Metabolic Diseases, Hannover Medical School, Hannover, Germany 
Hansen PR, Pediatric Nephrology, Hôpital Tivoli, La Louvière, Belgium

Jankauskiene A, Clinic of Children Diseases, Institute of Clinical Medicine, Vilnius University, Vilnius, Lithuania.

Jobs K, Department of Pediatrics, Pediatric Nephrology and Allergology, Military Institute of Medicine, Warsaw, Poland

Kopač M, Division of Pediatrics, Department of Nephrology, University Children's Hospital Ljubljana, Ljubljana, Slovenia

Liebau MC, Department of Pediatrics and Center for Molecular Medicine Cologne, University of Cologne, Faculty of Medicine and University Hospital Cologne, Cologne, Germany

Marks SD, Department of Paediatric Nephrology, Great Ormond Street Hospital for Children, NHS Foundation Trust, London, United Kingdom

Maxted A, Nottingham Children's Hospital, Nottingham, United Kingdom

Nalçacıoğlu H, Department of Pediatric Nephrology, Ondokuz Mayss University Faculty of Medicine, Samsun, Turkey

Oh J, Department of Pediatrics, University Medical Center Hamburg-Eppendorf, Hamburg, Germany

Özçelik G, Department of Pediatric Nephrology, Şişli Hamidiye Etfal Training and Research Hospital, İstanbul, Turkey

Papalia T, S.S. Nefrologia e Dialisi Pediatrica, Azienda Ospedaliera di Cosenza, Italy

Papizh S, Department of Hereditary and Acquired Kidney Diseases, Research and Clinical Institute for Pediatrics, Pirogov Russian National Research Medical University, Moscow, Russia

Poyrazoğlu H, Department of Pediatric Nephrology, Erciyes University Faculty of Medicine, Kayseri, Turkey

Prikhodina L, Department of Inherited and Acquired Kidney Diseases, Research and Clinical Institute for Pediatrics, Pirogov Russian National Research Medical University, Moscow, Russia

Schmidt IM, Department of Pediatrics and Adolescent Medicine, Copenhagen University Hospital, Copenhagen, Denmark

Schmitt CP, Center for Pediatrics and Adolescent Medicine, University Hospital Heidelberg, Heidelberg, Germany
Shroff R, Department of Paediatric Nephrology, Great Ormond Street Hospital for Children, NHS Foundation Trust, London, United Kingdom

Sönmez F, Department of Pediatric Nephrology, Adnan Menderes University Faculty of Medicine, Aydın, Turkey

Stabouli S, 1st Department of Pediatrics, School of Medicine, Faculty of Health Sciences, Aristotle University of Thessaloniki, Thessaloniki, Greece

Szczepanska M, Department of Pediatrics, Faculty of Medical Sciences in Zabrze, SUM in Katowice, Poland

Tabel Y, Department of Pediatric Nephrology, İnönü University Faculty of Medicine, Malatya, Turkey

Tasic V, Skopje University Children's Hospital, Skopje, Macedonia

Teixeira A, Pediatric Nephrology Unit, Centro Hospitalar do Porto, Porto, Portugal

Topaloğlu R, Department of Pediatric Nephrology, Hacettepe University Faculty of Medicine, Ankara, Turkey

Vande Walle J, Department of Pediatric Nephrology, University Hospital Ghent, Belgium

Vidal E, Department of Pediatrics, Udine University Hospital, Udine, Italy

Vondrak K, University Hospital Motol, Charles University Prague, Czech Republic

Yavaşcan Ö, Department of Pediatric Nephrology, Medipol University School of Medicine, İstanbul, Turkey

Yazıcıoğlu B, Department of Pediatric Nephrology, Gazi University Faculty of Medicine, Ankara, Turkey

Yildız G, Department of Pediatric Nephrology, Dokuz Eylül University Faculty of Medicine, İzmir, Turkey

Yilmaz D, Department of Pediatric Nephrology, Adnan Menderes University Faculty of Medicine, Aydın, Turkey

Zaloszyc A, Service de Pédiatrie 1, Hôpital de Hautepierre, Hôpitaux Universitaires de Strasbourg, Strasbourg, France

Zieg J, Department of Pediatrics, University Hospital Motol, Charles University Prague, Prague, Czech Republic

Publisher's note Springer Nature remains neutral with regard to jurisdictional claims in published maps and institutional affiliations. 